\title{
Turbulent Boundary Layers Over Multiscale Rough Patches
}

\author{
Christina Vanderwel $^{1}$ (D) Bharathram Ganapathisubramani ${ }^{1}$
}

Received: 6 April 2018 / Accepted: 10 January 2019 / Published online: 16 April 2019

(C) The Author(s) 2019

\begin{abstract}
We experimentally investigate the effects of multiscale rough patches on the drag and flow structure of a fully rough turbulent boundary layer in a wind tunnel. Several patches containing both organized and randomized arrangements of cubes of multiple sizes are tested in order to study the dependence of drag on the frontal solidity of the patch. The drag of each patch is measured with a drag balance for a range of Reynolds numbers, indicating a dependence of the drag on the frontal solidity following the trend predicted by Macdonald et al. (Atmos Environ 32(11):1857-1864, 1998). One of the patches is also replicated with the smallest scales removed and measurements show that the smaller scales have negligible impact on the overall drag. Flow fields in several cross-sections are captured using particle image velocimetry, and maps of the velocity deficit and increased turbulence activity in the wake of the patches are determined and used to define the extent of the internal boundary layer formed by each patch.
\end{abstract}

Keywords Roughness · Turbulent boundary layer · Urban meteorology

\section{Introduction}

Modelling of atmospheric flow over complex terrain is important for understanding the flow dynamics and corresponding dispersion in urban areas, as well as for the siting of wind farms in various onshore and offshore locations. Atmospheric boundary-layer (ABL) flow over complex terrain exhibits a range of characteristics with differing vertical profiles and high turbulence intensities. These variations are intimately related to the details of the surface terrain and the drag at the surface. Realistic surface terrain tends to be very rough, and roughness elements typically display a range of shape complexities and size distributions. It is important to be able to predict the flow features over such complex terrain for a range of applications; however, it is equally important to obtain experimental data (either in the field or through controlled wind-tunnel experiments) that can be used to validate the variety of modelling strategies. This paper presents results from a systematic series of experiments

Christina Vanderwel

c.m.vanderwel@soton.ac.uk

Bharathram Ganapathisubramani

g.bharath@soton.ac.uk

1 University of Southampton, Southampton, UK 
carried out in a wind tunnel to investigate the effect of the size distribution of roughness elements within a patch on the flow and drag incurred by that patch. We specifically focus on an isolated patch of multiscale roughness when the roughness elements attached to the surface are characterized by a broad size distribution, covering a large range of scales.

Previous investigations on rough-wall turbulent boundary layers have mostly focussed on regular roughness elements. Flow past an array of cubes has been studied extensively through observations, direct numerical simulation (DNS), as well as large-eddy simulation (LES) (see, e.g., Coceal et al. 2006; Castro et al. 2006). Based on measurements above cube arrays, Macdonald et al. (1998) assumed that fully-developed flow over a rough surface follows the logarithmic law,

$$
U^{+}=\frac{U}{u_{\tau}}=\frac{1}{\kappa} \ln \left(\frac{z-d}{z_{0}}\right),
$$

where $u_{\tau}$ is the friction velocity, $\kappa=0.38$ is the von Kármán constant, $z_{0}$ is the roughness length and $d$ is the zero-plane displacement. They then developed a relation between $z_{0}$, which is related to the aerodynamic drag of the surface, to the properties of the surface, specifically the frontal solidity $\lambda_{F}$, as

$$
\frac{z_{0}}{\langle H\rangle}=\left(1-\frac{d}{\langle H\rangle}\right) \exp \left(-0.5 \beta \frac{C_{D b}}{\kappa^{2}}\left(1-\frac{d}{\langle H\rangle}\right) \lambda_{F}^{-0.5}\right)
$$

where $\langle H\rangle$ is the average height of the cubes, $C_{D b}=1.2$ is the drag coefficient of an individual cube, and $\beta \approx 1.0$ is an adjustable coefficient. This relation has gained popularity for predicting the effects of surface roughness on the flow due to its simplicity, but it is not known how well it describes more complex surface roughness.

Most surfaces relevant to atmospheric flows are usually multiscale and comprise a range of different shapes and scales. Beside cubes, other shapes that have been considered include pyramids, ridges and more recently cuboids (Brown et al. 2001; Reynolds and Castro 2008; Schultz and Flack 2009; Castro et al. 2017). Recently, there is growing interest in understanding and predicting flows over multiscale surfaces. Multiscale roughness has also been examined for irregular roughness by Anderson and Meneveau (2011), who examined the flow over surfaces that have a power-law spectral distribution in height. Placidi and Ganapathisubramani $(2015,2018)$ examined flow past surfaces that contain LEGO bricks in different layouts where the brick was considered one scale while the pins on top of the bricks (and the baseboard) were a second scale/shape. Cheng and Castro (2002) carried out measurements over surfaces where repeated units had cuboids with a Gaussian height distribution and these were followed by LES studies of similar surfaces (Xie et al. 2008). More recently, Zhu et al. (2017) used LES to study the flow over surfaces with cuboids that have different mean heights and skewness distributions and used the data to develop a revised model for prediction of roughness length. Finally, Yang et al. (2016) examined flow past multiscale surfaces that consist of rectangular prisms, quadrupling the number of roughness elements on the ground as the sizes of the rectangular roughness elements were halved. As a result, the synthesized roughness followed a power-law height distribution.

In addition to consisting of a range of scales, terrain distributions in the natural environment typically vary along different geographic directions, for example from rural to urban regions, and the boundary layer has to adapt to this roughness transition. All of the above works focussed on homogeneous distributions of surface roughness (where a minimal unit is repeated along the streamwise and spanwise directions). There are a few numerical studies on turbulent flow over an explicitly-resolved roughness transition (Lee et al. 2011; Cheng 
and Porté-Agel 2015; Yang et al. 2016). In addition, experimental investigations are also scarce and typically only consist of one transition in surface roughness (Antonia and Luxton 1971; Cheng and Castro 2002; Hanson and Ganapathisubramani 2016). However, within complex terrain it is very likely that the distribution of roughness continuously changes, for example towards the centre of the city (or town). Such a patch of roughness is expected to exhibit similar qualities as abrupt changes in surface roughness, such as smooth-to-rough and rough-to-smooth transitions, which have been studied extensively. The effects of changing surface roughness are typically modelled by determining the evolution of the internal boundary layer, which is formed at the interface of the surface change, and which marks the boundary at which the effects of the surface no longer affect outer-layer similarity (Mahrt 2000; Bou-Zeid et al. 2004). When considering patches containing distinct obstacles, such as cubes or cylinders, the frontal and plan solidity (frontal area of the surface roughness and the plan area of the surface roughness per unit wall-parallel area) of the surface has been highlighted as an important parameter. In addition to solidity, the importance of the arrangement of the structures has also been highlighted, as this can produce "shielding" of downstream structures, where structures located in the wakes of other structures produce less drag (Yang et al. 2016). The solidity of a patch can also influence the vortex structures that develop in the wake and at the edges of the patch (Nepf 2012).

In this study, we bring together two of the above-mentioned lines of enquiry and examine the flow past a finite patch of multiscale cubes, concentrating on the effects of the patch arrangement on the drag and flow structure. We considered two different parameters: (i) the effect of frontal solidity, and (ii) the significance of the multiscale features. Firstly, we designed patches with equal planform solidities $\lambda_{P}$ (defined as the fraction of the planform area that is covered by cubes), but vary frontal solidity $\lambda_{F}$ (defined as the sum of forwardfacing faces normalized by the planform area, Grimmond and Oke 1999). In this way, for surfaces consisting of only cubes, $\lambda_{P}$ and $\lambda_{F}$ only differ if cubes are in contact, hence this difference is an indicator of shielding. Secondly, we replicated one of the patches with the smaller scales of cubes removed in order to evaluate the relative contribution of the different scales of roughness. The intent is to determine the minimum resolution required to properly resolve flow over complex features, which is important for computational studies.

\section{Design of the Patches}

Many features of nature exhibit fractal properties, including the height distribution of eroded surfaces and the layout of urban sprawl (Mandelbrot 1983). For this reason, our test cases are modelled using idealized fractal patterns. The designs of the multiscale patches are inspired by the Sierpinksi carpet fractal pattern, which is a plane fractal that comprises grids of squares of diminishing sizes. In order to create a three-dimensional (3D) patch of roughness from the Sierpinksi carpet, each square in the pattern is extruded into a cube. Four iterations of the fractal are included so that the patch includes four different sizes of cubes $(10,5,2.5$, $1.25 \mathrm{~mm}$ ). The number of each size of cube follows as near as possible to a power-law distribution (37, 140, 536, and 1884 cubes of each size, respectively) so the surface area covered by each scale is roughly equal. The patch is confined to a radius of $r=120 \mathrm{~mm}$ and 3D printed in polyamide resulting in a smooth texture and good resolution of even the smallest cubes. A 3D model of this patch is presented in Fig. 2, while planform views of this and the other patches in the study are presented in Fig. 1.

In the first part of the study, in order to investigate the effect of the arrangement of the cubes, in addition to the regular Sierpinksi pattern, four additional patches were constructed using 


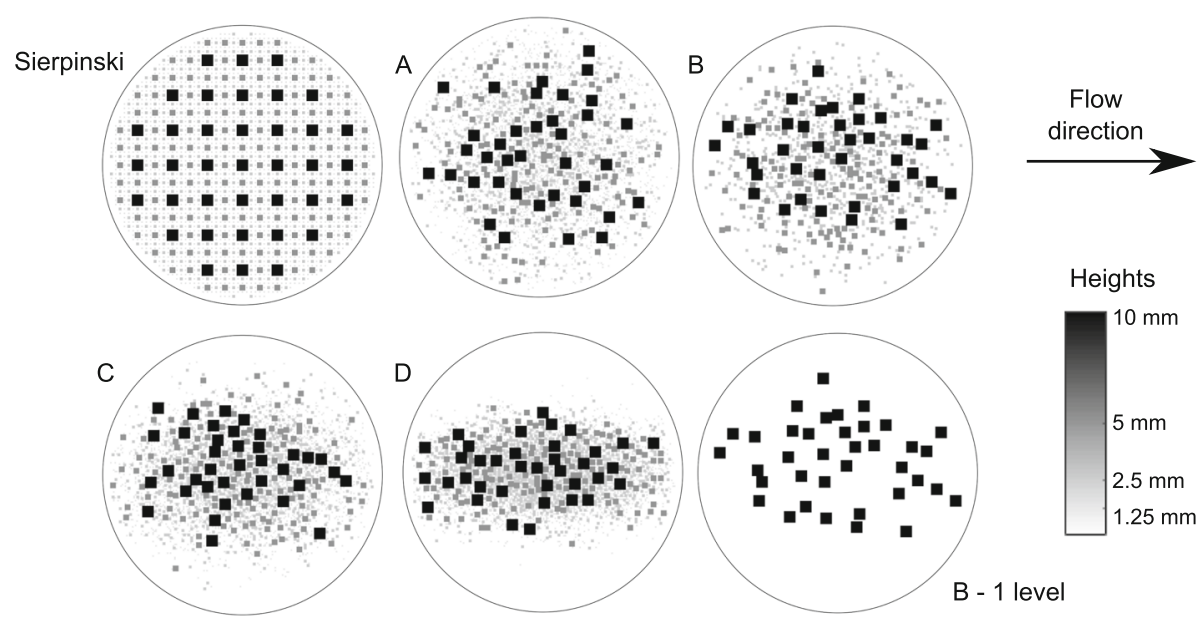

Fig. 1 The planform views of the different test patches
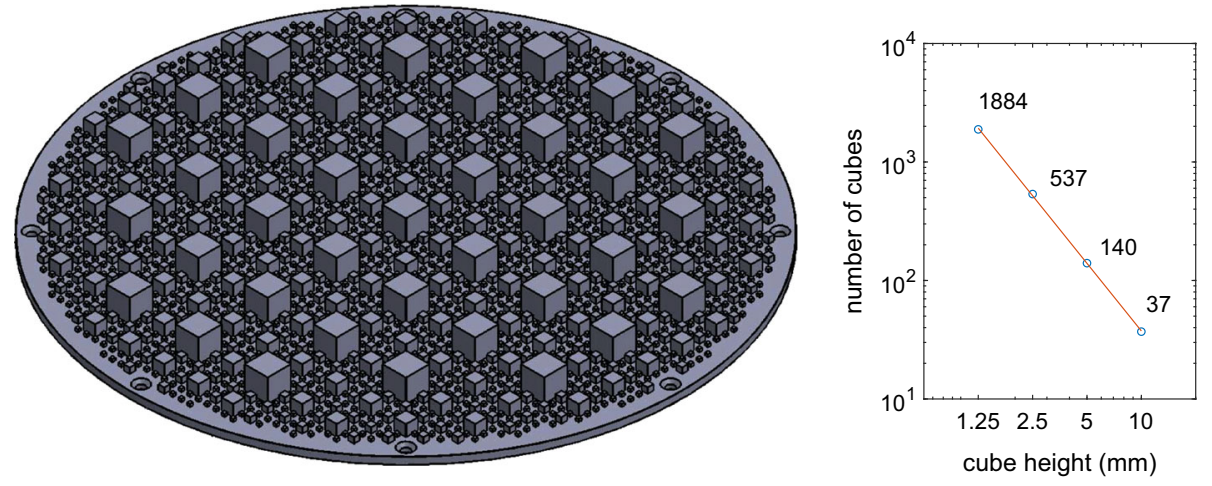

Fig. 2 (Left) The 3D model of the Sierpinski carpet patch. (Right) The histogram of the scales of cubes used in all of the layouts

random arrangements of the same number of cubes. The random distributions are created by randomly placing the cubes within the patch using a random number generator; however, in order to vary the frontal solidity $\lambda_{F}$ of each patch, we weighted the probability of placing a cube in a specific location by using a Gaussian distribution centred on the patch with varying $\sigma_{y}$, the second central moment of the arrangement in the spanwise direction. This varies how spread out the cubes are positioned and therefore the frontal solidity $\lambda_{F}$. As $\lambda_{F}$ is defined as the sum of forward-facing faces normalized by the planform area (Grimmond and Oke 1999), this represents the amount of "shielding" that occurs. The narrowest layout (layout D) has the fewest forward facing faces and therefore the smallest $\lambda_{F}$. Because the Sierpinski layout has no touching faces, it has the maximum value of $\lambda_{F}$. Because these patches all comprise the same number of cubes, each of these patches have the same planform solidity of $\lambda_{P}=0.298$. A histogram of the scales of cubes used in all of the layouts is presented in Fig. 2 resulting in an average obstacle height of $\langle H\rangle=1.84 \mathrm{~mm}$. Because this distribution of heights is the same for all layouts, all statistical parameters of this distribution are held constant, such as 
Table 1 Properties of the patches

\begin{tabular}{llll}
\hline Patch & $\sigma_{y} / r$ & $\lambda_{P}$ & $\lambda_{F}$ \\
\hline Sierpinski & 0.45 & 0.298 & 0.298 \\
Layout A & 0.38 & 0.298 & 0.254 \\
Layout B & 0.32 & 0.298 & 0.241 \\
Layout C & 0.27 & 0.298 & 0.232 \\
Layout D & 0.20 & 0.298 & 0.203 \\
B-1 level & 0.27 & 0.082 & 0.080 \\
\hline
\end{tabular}
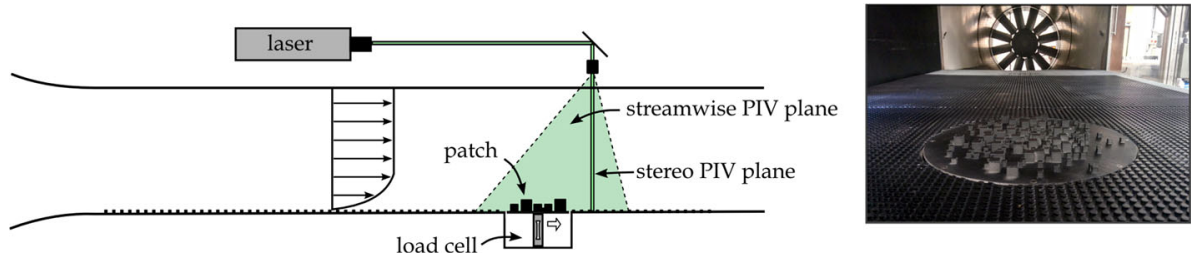

Fig. 3 Schematic of the experimental setup and a photograph of the patch installed in the wind tunnel

the height skewness, which can be an influential parameter (Zhu et al. 2017). Maps of the different arrangements are presented in Fig. 1 and their properties are shown in Table 1.

In the second part of the study, in order to investigate the contribution of the different scales, one of the randomized patches (layout B) is replicated with only the largest $10-\mathrm{mm}$ cubes included and all the smaller-scale cubes removed. In this way, we hope to decompose the contributions of the smaller scales to the total drag and flow structure of the patch. A map of the stripped-down patch is also presented in Fig. 1.

\section{Experimental Details}

Experiments were conducted in a suction wind tunnel at the University of Southampton; the test section is $4.5 \mathrm{~m}$ long and has a $0.9 \mathrm{~m} \times 0.6 \mathrm{~m}$ cross-section. A turbulent boundary layer was created on the floor of the tunnel, which was covered with homogeneous roughness created by LEGO baseboards made of bumps with a height of $1.7 \mathrm{~mm}$ (Placidi and Ganapathisubramani 2015; Vanderwel and Ganapathisubramani 2015). The patches were placed $4 \mathrm{~m}$ downstream of the leading edge of the baseboards where the boundary-layer thickness was $\delta=85 \mathrm{~mm}$, given a freestream velocity $U_{\infty}=20 \mathrm{~m} \mathrm{~s}^{-1}$. At this point, the incoming boundary layer was fully rough for all wind speeds considered here. As the maximum height of all patches was $H=10 \mathrm{~mm}$ this implies the patches were well-immersed in the boundary layer $(H / \delta=$ $0.12)$.

The drag of the patches was measured using a floating-element drag balance. The balance measured forces using multiple Tedea-Huntleigh model 1004 load sensors connected to a National Instruments PXIe-1062Q DAQ. The sensors were calibrated by hanging known weights on a string connected to the floating element through a pulley. The measurements were repeated three times for each patch and the uncertainty (including sensitivity and repeatability) of the load measurements was estimated to be $\pm 8 \times 10^{-4} \mathrm{~kg}$, which was an order of magnitude smaller than the magnitude of the measured drag.

The flow fields in two cross-sections of the flow were captured using conventional planar and stereoscopic particle image velocimetry (PIV) as shown in Fig. 3. Measurements in a 
streamwise vertical plane aligned with the patch centre were acquired using two side-by-side LaVision Imager Pro LX 16M cameras. The vector fields from the two cameras were then stitched together to create a long field-of-view that captured the incoming flow ahead of the patch and extended well into the wake of the patch. Subsequently, measurements of the patch wake were acquired in a plane located $63 \mathrm{~mm}$ downstream of the edge of the patch, using the same cameras placed in a stereoscopic configuration. For each case, 1000 image pairs were acquired at a rate of $0.7 \mathrm{~Hz}$. The light sheet was created by two synchronized Litron 200-15PIV Nd:YAG lasers that were in each case aligned using mirrors to form a single light sheet. The flow was seeded using a Magnum 1200 smoke machine. Vector fields were calculated using LaVision DaVis 8 using $32 \times 32$ pixel windows with a $50 \%$ overlap resulting in a vector spacing of roughly one vector $\mathrm{mm}^{-1}$ in both configurations. This linear resolution $(2 \mathrm{~mm}$ ) is just over the size of the smallest cube (which is $1.25 \mathrm{~mm}$ ). Therefore, the fluctuations of the order of that scale are not captured by the particle image velocimetry; however, our focus is on the larger scales of the flow and consequently this limitation in resolution is not considered to affect the results.

\section{Results: The Effect of Roughness Layout}

\subsection{Drag Measurements}

The drag of each patch was measured at four different wind speeds ranging from $U_{\infty}=$ 15 to $30 \mathrm{~m} \mathrm{~s}^{-1}$ and repeated three times for each case. Defining the Reynolds number as $R e_{L}=U L / v$, where $L$ is the fetch of the incoming boundary layer and $v$ is the kinematic viscosity of air, this results in $3.9 \times 10^{6}<\operatorname{Re}_{L}<7.7 \times 10^{6}$. The measurements are presented in Fig. 4a. The measured drag ranged between 0.020 and $0.035 \mathrm{~kg}$ at the top wind speed, and the differences between the drag of the different patches was significantly greater than the measurement uncertainty. For each patch, the measured drag increased with wind speed following a power law as $F_{D}=a \operatorname{Re}_{L}{ }^{n}$, where $n=2.0 \pm 0.1$ as would be expected if the drag coefficient is constant. The drag coefficient of each patch is defined as

$$
C_{D}=\frac{F_{D}}{0.5 \rho U_{\infty}^{2} A_{P}}
$$

(a)

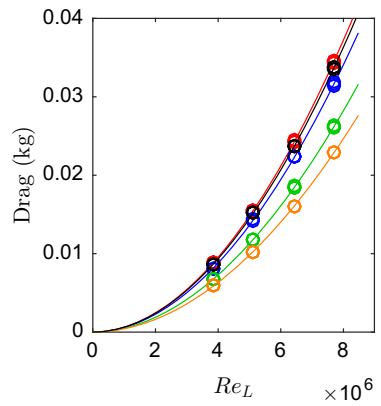

(b)

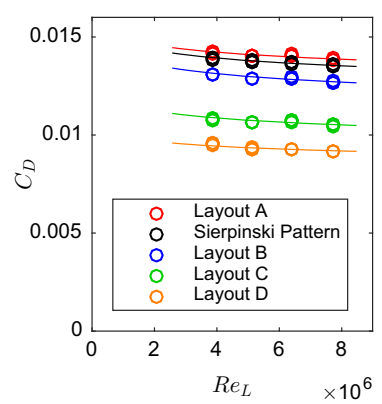

(c)

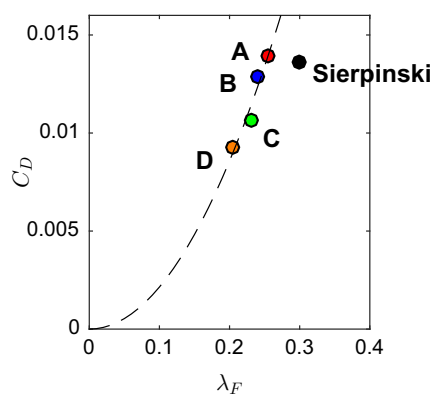

Fig. 4 a Drag measurements of the five patches. b The corresponding drag coefficients. c Average values of $C_{D}$ for each patch versus the patch's frontal solidity $\lambda_{F}$, with the fit of Eq. 4 based on Macdonald et al. (1998) shown as a dashed line 
where $A_{P}=\pi r^{2}$ is the planform area of the patch. The values of $C_{D}$ are presented in Fig. $4 \mathrm{~b}$ and were nearly constant for each case, varying by less than $3 \%$ over the range of tested Reynolds numbers. This suggests that the flow phenomena do not drastically change with Reynolds number. This is typical of turbulent flows around features with sharp edges such as cubes, as the sharp edge fixes the separation point; a laminar flow, on the other hand, might produce vortex shedding that is sensitive to the wind speed.

Comparing the drag measurements of the different randomized arrangements, the results show a monotonic increase in drag with increasing frontal solidity in Fig. 4c.

In a fully-developed boundary-layer, the roughness length $z_{0}$ from the logarithmic-law fit of Eq. 1 can be directly related to the momentum deficit, and hence the overall drag of the surface (Macdonald et al. 1998). In fact, the value of $z_{0}$ is often used as a surrogate to drag measurements, especially in numeral studies (Yang et al. 2016; Zhu et al. 2017). Because the present flow is not fully-developed over the patch, such a direct relation cannot be made; however, we can still check if the expected relationship between the drag and the surface property $\lambda_{F}$ proposed by Macdonald et al. (1998) in Eq. 2 is consistent with the present results. Adapting this relationship, assuming that the drag coefficient will still be somehow proportional to the predicted $z_{0}$ of Macdonald et al., we would expect a relationship of the form

$$
C_{D}=\alpha \exp \left(-\left(\gamma \lambda_{F}\right)^{-0.5}\right) .
$$

We find that this relationship does indeed describe well the present results, given values of $\alpha=0.54$ and $\gamma=0.29$, which were determined from a best-fit to the present data. This relation is also plotted in Fig. $4 \mathrm{c}$ showing an excellent fit.

This increase in drag with frontal solidity is consistent with the idea that the "shielding" of downstream elements, which reduces the frontal solidity of a surface, can significantly reduce drag. What is interesting is that the drag of the orderly Sierpinski carpet arrangement, which technically has the highest frontal solidity, produced a drag comparable to layout A, which had a lower $\lambda_{F}$ value although the highest of the randomized arrangements. This suggests that frontal solidity alone is insufficient to predict drag, especially when the surface structure is organized in some way rather than randomized. In the special case of the Sierpinksi carpet arrangement, although no cubes are in contact, many lie in the wakes of others increasing the effective amount of "sheilding". Furthermore, many alleyways are left exposed for air to flow through, making the flow act in a similar way to a patch with less solidity.

\subsection{Velocity Fields}

The velocity fields were captured in two cross-sections of the flow for each patch, with a freestream velocity $U_{\infty}=20 \mathrm{~m} \mathrm{~s}^{-1}\left(\operatorname{Re}_{L}=5.1 \times 10^{6}\right)$. As the drag coefficients of each patch are fairly independent of wind speed, we expect the flow patterns measured at this speed to be representative. The velocity maps confirm that the incoming boundary layer has a thickness of $\delta=85 \mathrm{~mm}$. Examples of typical instantaneous velocity maps acquired in both measurement planes are presented in Fig. 5.

\subsubsection{The Incoming Flow}

The flow that impinged upon the roughness patches was a fully developed rough-wall turbulent boundary-layer flow that developed over the rough-wall LEGO baseboard that covered the test section floor. Measurements were collected over the same field of view as shown 


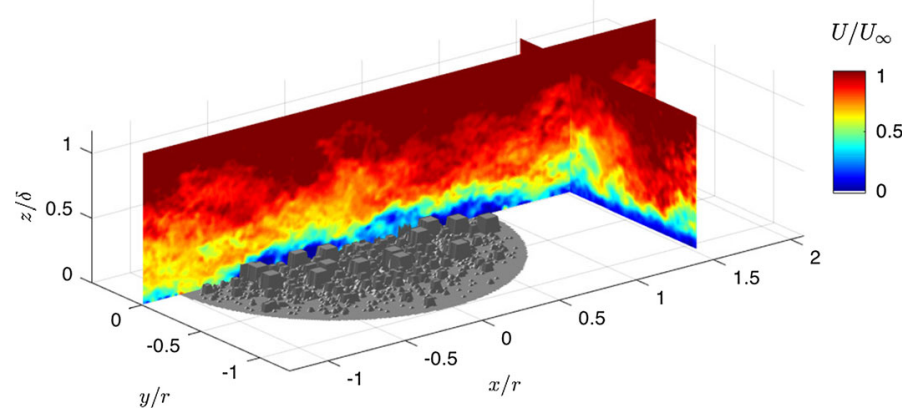

Fig. 5 Examples of the instantaneous velocity fields over patch B

(a)

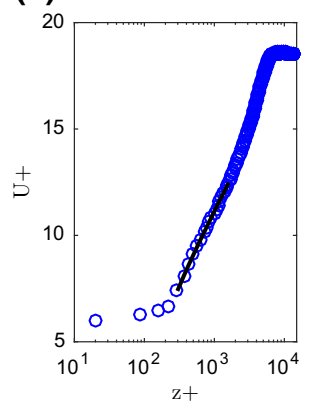

(b)

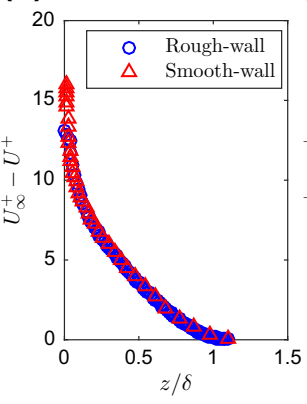

(c)

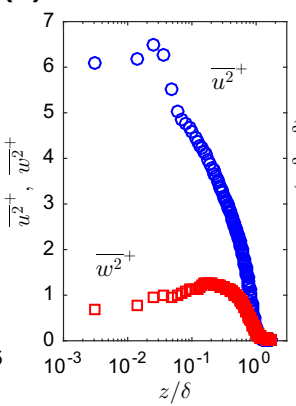

(d)

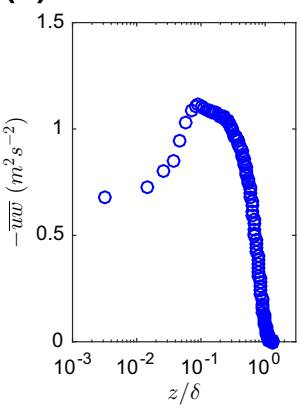

Fig. 6 a Mean incoming velocity profile over the LEGO baseboard rough-wall, normalized by inner units indicating the log-law region. $\mathbf{b}$ The mean velocity profile of the incoming rough-wall flow in velocity-defect form, compared to a smooth-wall case. $\mathbf{c}$ The profile of streamwise and vertical velocity fluctuation intensities of the incoming rough-wall flow. d The mean Reynolds shear stress of the incoming rough-wall flow

in Fig. 5, but with a continuation of the LEGO baseboard replacing the patch in order to confirm that the flow was no longer significantly developing in the streamwise direction. The representative velocity profile of this incoming flow and some second-order statistics are presented in Fig. 6 . The friction velocity, $u_{\tau}$, was determined using two methods. The first method assumes mean flow similarity and that the mean velocity profile in velocitydefect form should collapse with smooth-wall data (Flack and Schultz 2010). The velocity profile is plotted in velocity-defect form in Fig. $6 \mathrm{~b}$ and compared with smooth-wall data that was previously collected via hot-wire anemometry in the same facility by Takfarinas Medjnoun. A value of $u_{\tau} \approx 1.05 \mathrm{~m} \mathrm{~s}^{-1}$ leads to a collapse of the velocity-deficit data with the smooth-wall data. A second method to estimate $u_{\tau}$ is from the near-wall peak of the averaged Reynolds shear stresses (Reynolds and Castro 2008; Placidi and Ganapathisubramani 2015). These are plotted in Fig. 6c, which indicate the peak of the shear stress reaches approximately $1.05 \mathrm{~m}^{2} \mathrm{~s}^{-2}$. Using the correction factor proposed by Reynolds and Castro (2008), this corresponds to $u_{\tau}=1.12 \sqrt{\overline{-u w_{\text {peak }}}}=1.15 \mathrm{~m} \mathrm{~s}^{-1}$. In Fig. $6 \mathrm{c}$, the profiles of streamwise and vertical velocity fluctuation intensities, normalized by an average value of $u_{\tau}=1.1$, is consistent with earlier data (Squire et al. 2016). With the friction velocity known, the mean velocity profile can be fit to the expected logarithmic-law profile for fully-rough turbulent boundary layers given in Eq. 1. For the incoming flow we found $z_{0}=0.2 \mathrm{~mm}$ and $d=0.8 \mathrm{~mm}$, which is consistent with what we would expect for the flow above the 
LEGO baseboard (Placidi and Ganapathisubramani 2015). Figure 6a presents the velocity profile, normalized by inner units, showing that the logarithmic region extends roughly over the range $4 \mathrm{~mm}<z<20 \mathrm{~mm}$, which means that the height of the patches $(H=10 \mathrm{~mm})$ is well-immersed in the logarithmic region of the incoming boundary layer.

\subsubsection{Visualizing the Internal Boundary Layer Over the Patch}

In order to distinguish the impact of the patch, the average velocity deficit in the vicinity of the patch is computed as

$$
\Delta U=\bar{U}-\bar{U}_{\text {ref }}
$$

where $\bar{U}$ is the average velocity field and $\bar{U}_{\text {ref }}$ is the velocity map measured using the rough base without any patch. Figure 7a, e show example maps of this velocity deficit for patch $\mathrm{B}$ and the Sierpinksi patch, which have similar total drag but differently shaped wakes. The internal boundary layer created by the patch is defined as the contour line corresponding with a velocity deficit of $\Delta U / U_{\infty}=1 \%$. For all the patches, the internal boundary layer does not extend beyond $4 \mathrm{H}$ from the floor, indicating that the patches does not affect similarity in the outer region of the boundary layer. The exception to this is a relatively large region at the leading edge of the patch due to the initial deflection of the flow around the patch, but which does not seem to influence the wake that forms downstream. In each case, the wakes extend several radii downstream. The length of the wake, $L_{W}$, is defined as the distance from the trailing edge of the patch to the point where the velocity deficit in the wake recovered to $10 \%$ of $U_{\infty}$. Results are summarized in Table 2. In general, the wakes of the narrower patches extend farther downstream, with the wake of the narrowest patch (layout D) extending roughly $20 \%$ farther than that of the widest patch (layout A). This indicates that, although the wider patches create more drag, the denser narrower patches create more focused disturbances to the flow that take longer to recover.

To visualize the impact of each patch on the turbulent kinetic energy in the flow, the additional root-mean-squared streamwise velocity fluctuation created by the patch is defined as

$$
\Delta u_{\mathrm{rms}}=u_{\mathrm{rms}}-\left(u_{\mathrm{rms}}\right)_{\mathrm{ref}}
$$

where $u_{\mathrm{rms}}$ denotes the root-mean-square streamwise velocity fluctuations and $\left(u_{\mathrm{rms}}\right)_{\mathrm{ref}}$ is the background value of $u_{\mathrm{rms}}$ present without any patch installed. Example maps are presented in Fig. 7b, f. Similarly, the additional root-mean-squared vertical velocity fluctuation is denoted as $v_{\text {rms }}$ and presented in Fig. 7c, g. The results indicate increased turbulent activity in the wake of each patch compared with having no patch, the extent of which is consistent with the edge of the internal boundary layer identified by the velocity deficit. Similarly, to visualize the impact of each patch on the Reynolds shear stress in the flow, the additional stress created by the patch was computed and is presented in Fig. $7 \mathrm{~d}$, $\mathrm{h}$ and has an extent consistent with the edge of the internal boundary layer.

\subsubsection{Investigating the Wake}

As a second indication of the drag of the patch, the momentum deficit in the spanwise crosssection located downstream of the patch is calculated as

$$
\Delta M=\rho \iint \bar{U}\left(\bar{U}_{\text {ref }}-\bar{U}\right) \mathrm{d} y \mathrm{~d} z .
$$


Patch B
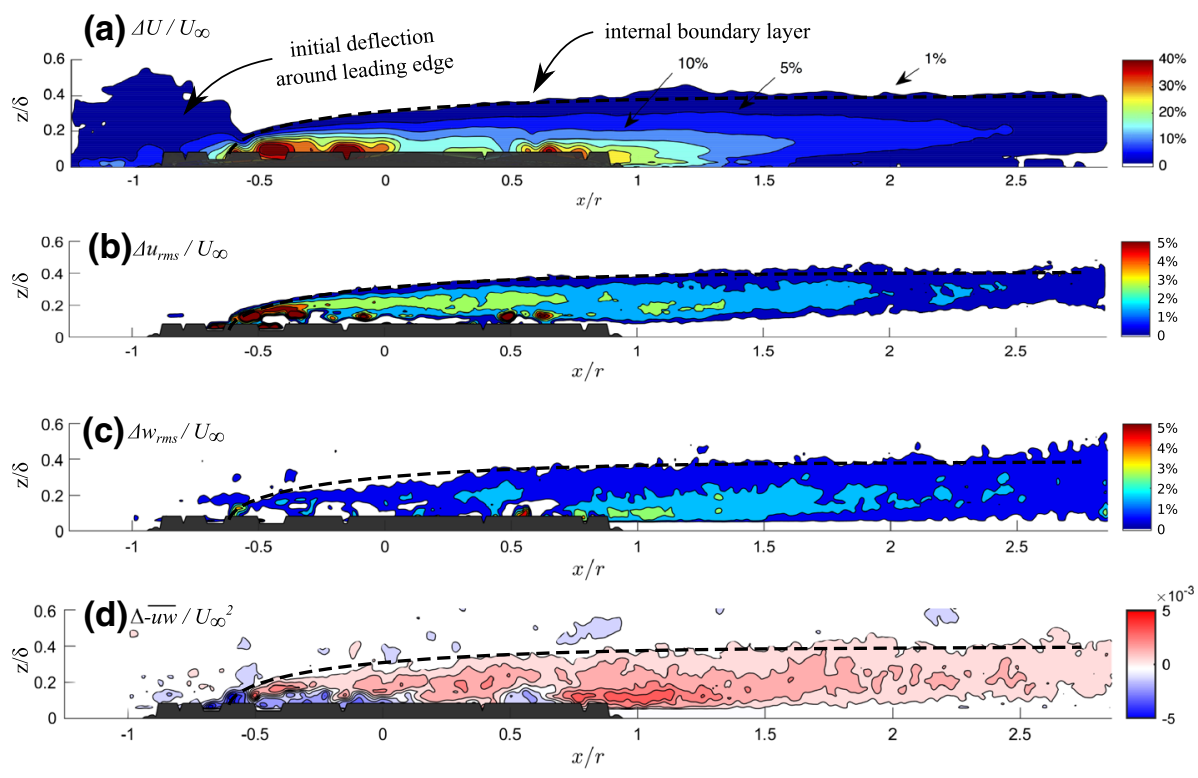

(e) $\Delta U / U_{\infty}$

Patch Sierpinski
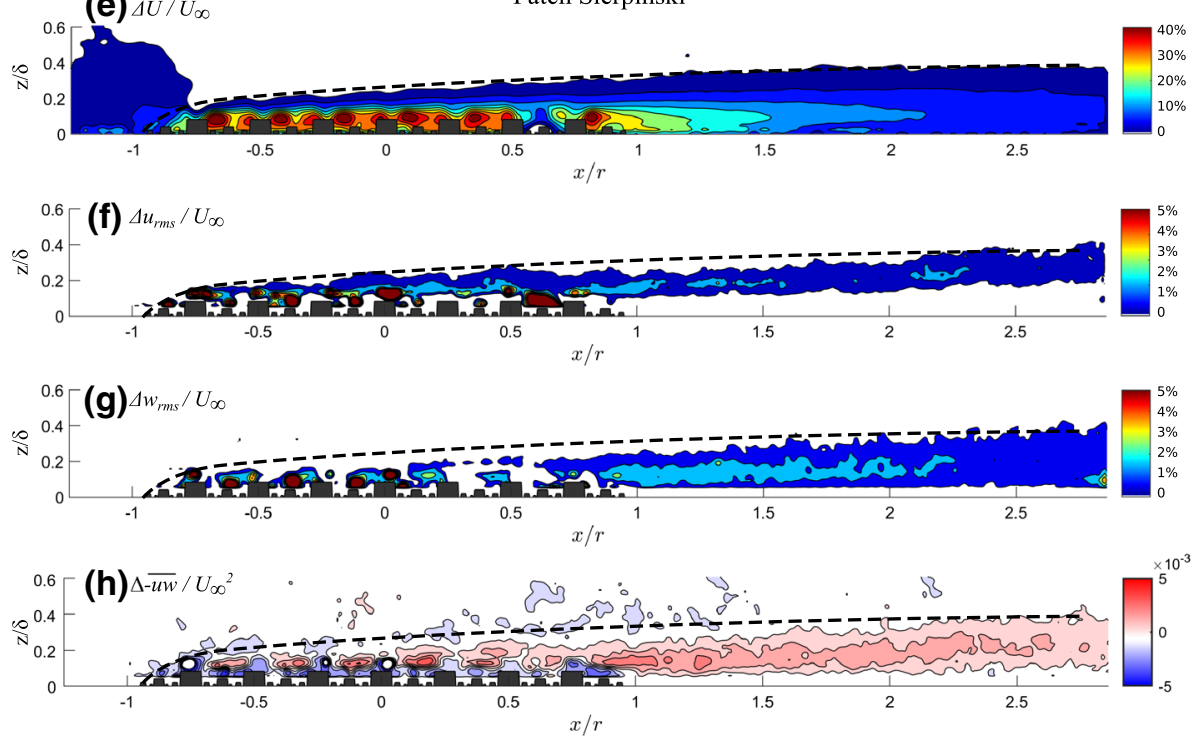

Fig. 7 Representative measurements over patch B and the Sierpinksi patch. a, e Maps of the average velocity deficit $\Delta U$ created by the presence of the patch when compared with the flow field with no patch. The $1 \%$ contour identifies the edge of the internal boundary layer, with the exception of a localized region around the leading edge of the patch where the flow initially deflects around the patch. $\mathbf{b}$, $\mathbf{f}$ Maps of the average additional streamwise velocity fluctuation intensities around the patch. $\mathbf{c}$, $\mathbf{g}$ Maps of the average additional vertical velocity fluctuation intensities around the patch. In $\mathbf{b}, \mathbf{c}, \mathbf{f}, \mathbf{g}$, the $1 \%$ contour coincides with the edge of the internal boundary layer. $\mathbf{d}, \mathbf{h}$ Maps of the average additional Reynolds shear stress around the patch 
Table 2 Measurements of the wakes of the patches obtained at $R e_{L}=5.1 \times 10^{6}$ : the drag balance measurement, $F_{D}$, the momentum deficit, $\Delta M$, converted to $\mathrm{kg}$, the lengths of the wakes, $L_{W}$, along with the calculated $u_{\tau}$ and the values of $z_{0}$ and $d$ determined from a best fit to the velocity profiles in the wakes of the patches at $x=1.5 r$, spanwise-averaged over $-r<y<r$

\begin{tabular}{llllllll}
\hline Patch & $\lambda_{F}$ & $F_{D}(\mathrm{~kg})$ & $\Delta M(\mathrm{~kg})$ & $L_{W} / r$ & $u_{\tau}\left(\mathrm{m} \mathrm{s}^{-1}\right)$ & $z_{0}(\mathrm{~mm})$ & $d(\mathrm{~mm})$ \\
\hline Sierpinski & 0.298 & $15.3 \times 10^{-3}$ & $5.6 \times 10^{-3}$ & 2.13 & 1.65 & 1.14 & 2.3 \\
Layout A & 0.254 & $15.6 \times 10^{-3}$ & $6.4 \times 10^{-3}$ & 1.83 & 1.67 & 1.18 & 2.5 \\
Layout B & 0.241 & $14.4 \times 10^{-3}$ & $6.1 \times 10^{-3}$ & 1.63 & 1.60 & 1.00 & 2.4 \\
Layout C & 0.232 & $11.9 \times 10^{-3}$ & $4.2 \times 10^{-3}$ & 2.08 & 1.46 & 0.70 & 3.0 \\
Layout D & 0.203 & $10.3 \times 10^{-3}$ & $3.7 \times 10^{-3}$ & 2.19 & 1.36 & 0.52 & 2.9 \\
\hline
\end{tabular}

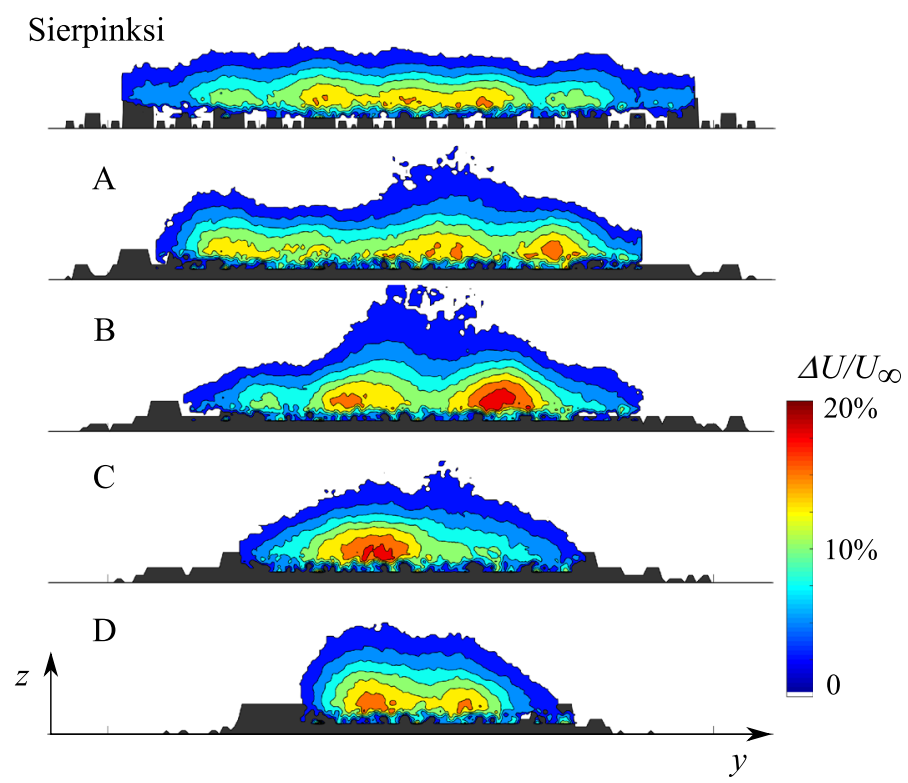

Fig. 8 Maps of the average velocity deficit downstream of each of the patches

Maps of the average velocity deficit downstream of each of the patches are presented in Fig. 8 and the calculated momentum deficits are presented in Table 2. When converted into the equivalent kilogram force, it is apparent that the momentum deficit only accounts for a fraction of the total drag measured by the drag balance; however, this discrepancy is a result of the fact that the measurement plane is in the near field where the pressure would not have fully recovered to the upstream value. Nevertheless, the momentum deficits follow similar trends as the drag measurements: the wider patches created larger wakes and therefore more drag. Once again, the exception is the Sierpinksi pattern, which has less momentum deficit and a longer wake than the randomized layouts with similar solidity. While the alleyways left open in the regular Siepinski layout result in a weaker wake and hence less momentum drag, they must also not be conducive to the kinds of flow structures that help a wake recover, resulting in a longer wake. This highlights the fact that organized arrangements cannot be treated in the same general manner as randomized surfaces. 

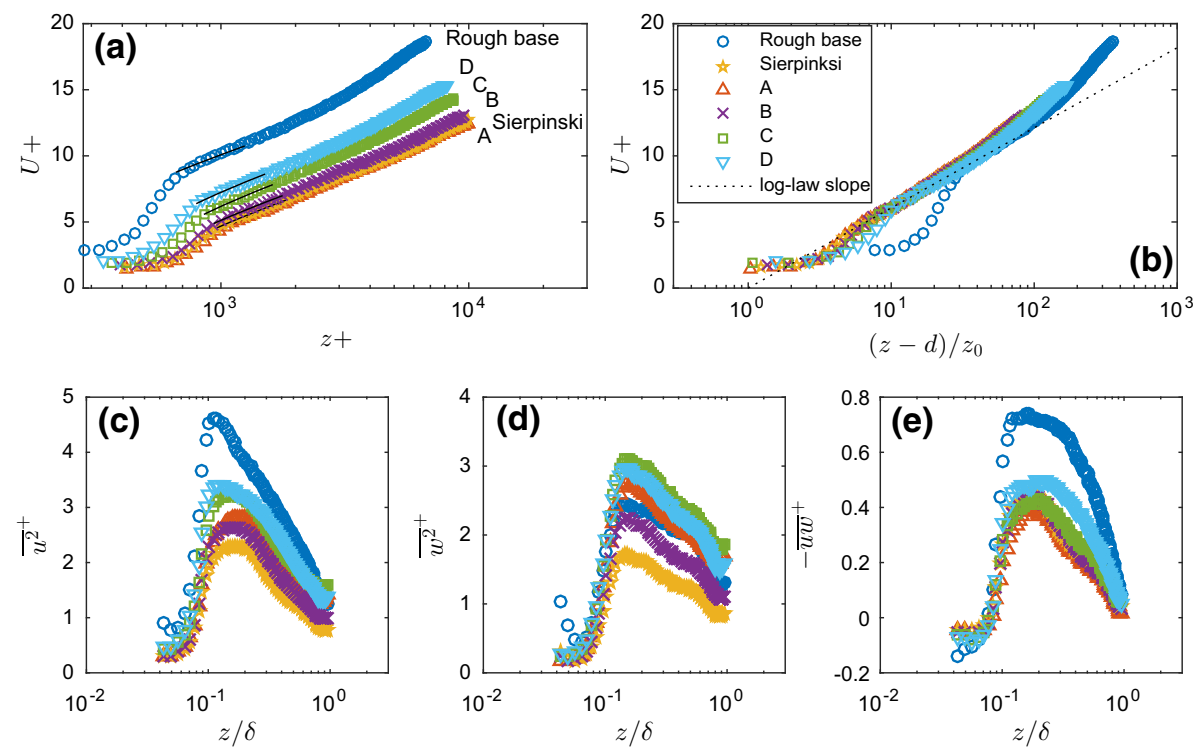

Fig. 9 Velocity profiles and statistics in the wakes of the patches at $x=1.5 r$, spanwise-averaged over $-r<y<r$. a Plots normalized by inner units show the offsets in the profiles corresponding to different $z_{0}$ values. b Plots normalized by $z_{0}$ show a collapse in the log region of the boundary layer, although the outer wake region lacks similarity with this scaling and the extent of the near-wall region varies with $d$. c-e Plots of the velocity statistics also normalized by inner units exhibit similar shapes between all the patches, but vary in magnitude following the same order as the values of $u_{\tau}$ of each patch

Another indication of the drag of the patch is the value of the roughness length $z_{0}$ of the modified velocity profile, which is often used as a surrogate to drag measurements (Macdonald et al. 1998; Yang et al. 2016; Zhu et al. 2017). In a fully-developed boundary layer, the value of $z_{0}$ can be related to the momentum deficit, and hence the drag, by integrating the velocity profile. With a developing flow, such a direct relation cannot be made, and this is especially true for any measurements located over the patch, as they would surely vary with the spanwise surface heterogeneity. However, we can investigate whether the velocity fields in the wake of the patch, where we can spanwise-average across $-r<y<r$ to alleviate any issues with spanwise heterogeneity, have recovered any self-similarity and whether an increase in aerodynamic drag can still be correlated with an increase in $z_{0}$. To this end, and to accommodate comparisons with other studies, we have plotted the velocity profiles and statistics in the wakes of the patches at $x=1.5 r$, spanwise-averaged over $-r<y<r$ in Fig. 9 and determined modified logarithmic-law fits for each profile. The friction velocities for each profile were determined from the measured drag coefficient (presented in Fig. 4) through the relation $C_{D}=2\left(u_{\tau} / U_{\infty}\right)^{2}$. Subsequently, the effective roughness length $z_{0}$ and displacement height $d$ were determined using a best fit. These parameters are presented in Table 2 for each individual patch. The displacement height $d$ does not vary significantly for the different patches, which is expected as the height distribution of the cubes is the same among all patches. The roughness length $z_{0}$ follows a similar trend to the $C_{D}$ measurements, increasing with frontal solidity $\lambda_{F}$, again with the exception of the organized Sierpinski patch.

As shown in Fig. 9, the velocity profiles and statistics do not completely collapse when normalized by inner units, which can be attributed to the fact that, at this position in the wake 
of the patch, this is not a fully-developed flow, so $u_{\tau}$ does not fully dictate the boundarylayer characteristics. When the velocity profiles in the wake are normalized by the roughness length, as shown in shown in Fig. 9b, they indicate a collapse in the logarithmic region; however, the outer wake region lacks similarity with this scaling. This is not unexpected as we showed earlier that the inner boundary layer formed by the patch does not extend beyond $z>0.5 \delta$.

We found that if this logarithmic fit was performed with velocity data measured in the flow developing over the patch, the calculated values of $z_{0}$ differ by as much as $100 \%$ due to the lack of local equilibrium. Therefore, we caution using $z_{0}$ from arbitrary measurement locations as a surrogate for drag over patches, as the flow is clearly not fully-developed everywhere, manifesting in significant variability in the local boundary layer and uncertainty in the logarithmic-law fits.

\section{Results: Significance of the Small Scales}

\subsection{Drag Measurements}

The drag measurements of layout B replicated with only the 10 - $\mathrm{mm}$ cube features are presented in Fig. 10. The 10-mm cube features represent roughly one quarter of the surface area that was originally covered by obstacles, while the other three-quarters of the covered area previously had obstacles of $5,2.5$, or $1.25 \mathrm{~mm}$. The difference in the drag of the complete patch and the one containing only the largest size of cubes was only approximately $5 \%$, indicating that the addition of the smaller scales appears to be minimal.

The drag was also measured without the patch installed in order to estimate the contributions of the pressure drag and the additional skin friction from the cubes. As shown in Fig. 1, the drag with no patch and just a continuation of the rough surface was roughly half the magnitude of those of the patches, demonstrating that the presence of the cubes was indeed significant. The drag of a smooth patch was also measured for comparison, and was found to be half again of the drag of the rough surface.

Considering these different cases, we can attempt to decompose the various sources of drag. The drag of the smooth surface was only $18 \%$ of the value of the total drag of Layout B. When the largest cubes were added, the measured drag was $96 \%$ of the final value, indicating

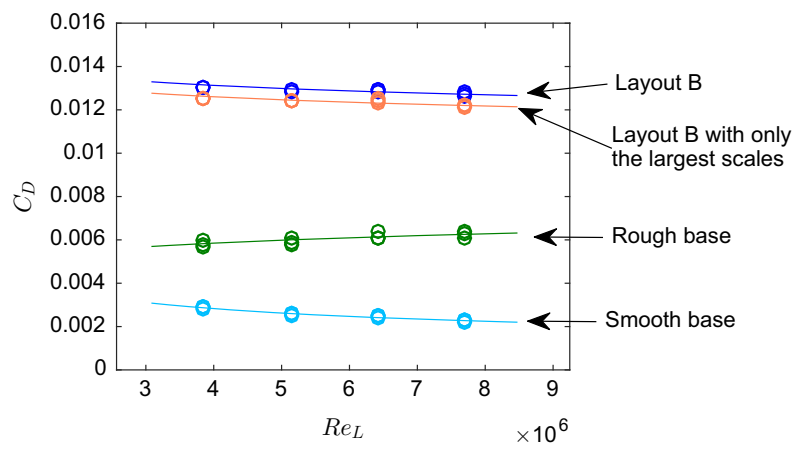

Fig. 10 Measurements of the drag coefficients of layout B, layout B replicated with only the largest 10-mm cubes, the patch replaced with a continuation of the homogeneous roughness in the rest of the wind-tunnel, and the patch replaced with a smooth plate 


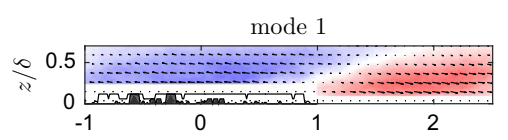

mode 2

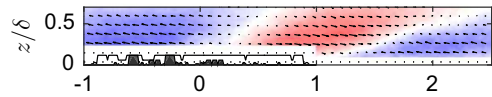

mode 3

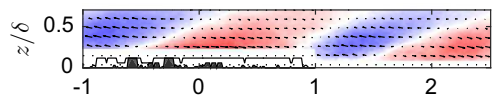

mode 4

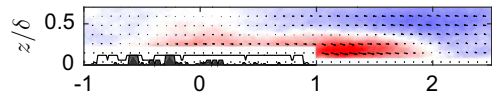

mode 5

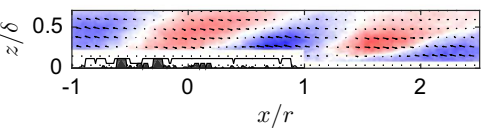

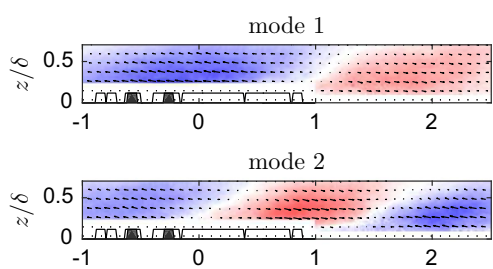

mode 3

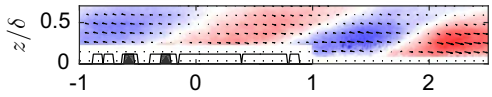

mode 4

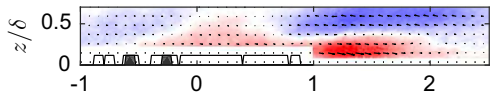

mode 5

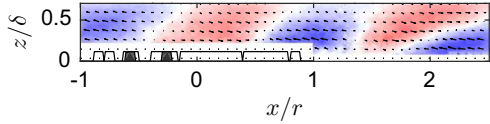

Fig. 11 Side-by-side comparison of the first five most dominant POD modes in the flow around (left) the full layout B patch and (right) layout B stripped-down to include only the largest-scale cubes

that these scales are responsible for the majority, $78 \%$, of the total drag. This implies that the three smaller scales of cubes only contributed about $4 \%$ of the total drag. It is worth noting that although the three smaller scales of cubes originally represented the majority of the area covered by obstacles, they are at least half the height of the large 10-mm cubes, and this scale-separation would be important to neglect the smallest scales. This simplistic decomposition is particularly crude as it assumes that the effects of each scale of cube are cumulative and neglects any coupling (such as shielding) that occurs. However, the fact that the measurements did not differ significantly is good news for computational modellers, as this suggests that a very fine resolution of the surface texture features is not necessary to get reasonable predictions of the drag.

\subsection{Velocity Fields}

The averaged velocity fields also showed that the wakes of the patch with different numbers of cubes were essentially indiscernible. To further investigate if the dominant instantaneous flow features are also statistically identical, we performed a proper orthogonal decomposition (POD) analysis on the instantaneous snapshots. POD identifies the basis modes that make up a turbulent flow field and is a robust way of analyzing whether the significant features of the flow field have changed beyond simply the aggregate measures. The spatial resolution of PIV can be expected to affect the analysis carried out using POD; however, here, the focus is on the comparison between the large-scale most energetic modes across different surfaces and the spatial resolution of the PIV is maintained for all our surfaces at $150 \times$ 150 wall-units (interrogation window size) relative to the incoming flow properties. Placidi and Ganapathisubramani (2018) found that, for an urban canopy flow at this resolution, approximately $30 \%$ of the energy is held in the first five modes and all these modes remain similar across different surface morphologies. Here, we can examine if an isolated patch alters this finding. The five most energetic modes from the flows around the full and stripped-down patches are presented in Fig. 11. These modes are clearly identical for these two flows, and 

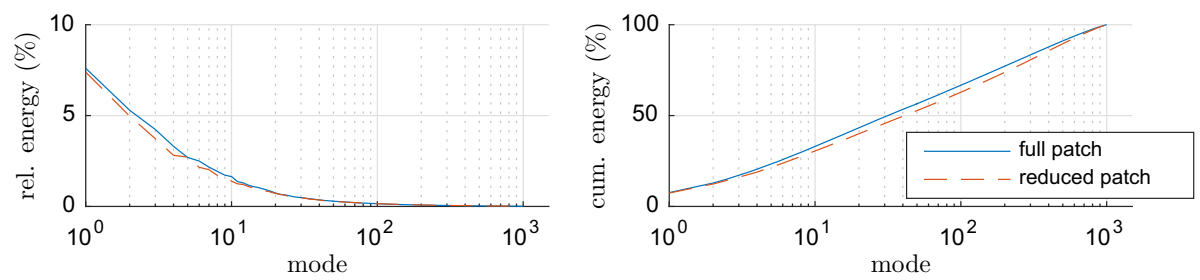

Fig. 12 Comparison of the relative and cumulative energy distributions of the POD modes in the flow around the full layout B patch and reduced layout B stripped-down to include only the largest-scale cubes

show that the dominant fluctuations in the flow appear to stem from the base of the patch and from the locations of the largest cubes, which are consistent between the two patches. The relative energy content of all the modes is shown in Fig. 12 and was also nearly identical. This suggests that the large-scale energy distribution in the flow is not affected by the presence of the finer scales in the surface topography.

This demonstrates that only the largest scales of the patches are important for dictating these integral properties of the flow, such as the turbulent kinetic energy and drag. It is not yet known whether this would also be true for processes that depend more on the smallscale contributions, such as mixing, and further studies are needed to investigate this directly. Further investigation would be required to investigate under which conditions this is also true for arbitrary rough patches.

\section{Conclusions}

Measurements of the drag and flow structure above finite multiscale patches showed that, in all cases, the internal boundary layers created by the patches were much shallower than the boundary-layer thickness, indicating that the patches did not affect outer-layer similarity. The results indicate that the drag of the randomized patches increase monotonically with increasing frontal solidity, illustrating how "shielding" reduces drag. Furthermore, we showed that the majority of the drag is attributed to the largest scales of roughness. We also found that the patch that was organized as a Sierpinski pattern did not fit the same trend as the randomized arrangements, highlighting how exposed alleyways through the patch significantly affect the flow.

Acknowledgements We gratefully acknowledge the support of the H2020 Marie Sklodowsa-Curie European Fellowship and EPSRC (Grant Ref No. EP/P021476/1). We also thank our summer intern Mantas Gudaitis for his contribution to the data analysis. All data supporting this study are openly available from the University of Southampton repository at https://doi.org/10.5258/SOTON/D0769.

Open Access This article is distributed under the terms of the Creative Commons Attribution 4.0 International License (http://creativecommons.org/licenses/by/4.0/), which permits unrestricted use, distribution, and reproduction in any medium, provided you give appropriate credit to the original author(s) and the source, provide a link to the Creative Commons license, and indicate if changes were made.

\section{References}

Anderson W, Meneveau C (2011) Dynamic roughness model for large-eddy simulation of turbulent flow over multiscale, fractal-like rough surfaces. J Fluid Mech 679:288-314 
Antonia RA, Luxton RE (1971) The response of a turbulent boundary layer to a step change in surface roughness part 1. Smooth to rough. J Fluid Mech 48(04):721-761

Bou-Zeid E, Meneveau C, Parlange MB (2004) Large-eddy simulation of neutral atmospheric boundary layer flow over heterogeneous surfaces: blending height and effective surface roughness. Water Resour Res. https://doi.org/10.1029/2003WR002475

Brown AR, Hobson J, Wood N (2001) Large-eddy simulation of neutral turbulent flow over rough sinusoidal ridges. Boundary-Layer Meteorol 98(3):411-441

Castro IP, Cheng H, Reynolds R (2006) Turbulence over urban-type roughness: deductions from wind-tunnel measurements. Boundary-Layer Meteorol 118(1):109-131

Castro IP, Xie ZT, Fuka V, Robins AG, Carpentieri M, Hayden P, Hertwig D, Coceal O (2017) Measurements and computations of flow in an urban street system. Boundary-Layer Meteorol 162(2):207-230

Cheng H, Castro IP (2002) Near wall flow over urban-like roughness. Boundary-Layer Meteorol 105:411-432

Cheng W, Porté-Agel F (2015) Adjustment of turbulent boundary-layer flow to idealized urban surfaces: a large-eddy simulation study. Boundary-Layer Meteorol 155(2):249-270

Coceal O, Thomas T, Castro I, Belcher S (2006) Mean flow and turbulence statistics over groups of urban-like cubical obstacles. Boundary-Layer Meteorol 121(3):491-519

Flack KA, Schultz MP (2010) Review of hydraulic roughness scales in the fully rough regime. J Fluids Eng 132(4):041203

Grimmond CSB, Oke TR (1999) Aerodynamic properties of urban areas derived from analysis of surface form. J Appl Meteorol 38(9):1262-1292

Hanson R, Ganapathisubramani B (2016) Development of turbulent boundary layers past a step change in wall roughness. J Fluid Mech 795:494-523

Lee JH, Sung HJ, Krogstad PÅ (2011) Direct numerical simulation of the turbulent boundary layer over a cube-roughened wall. J Fluid Mech 669:397-431

Macdonald R, Griffiths R, Hall D (1998) An improved method for the estimation of surface roughness of obstacle arrays. Atmos Environ 32(11):1857-1864

Mahrt L (2000) Surface heterogeneity and vertical structure of the boundary layer. Boundary-Layer Meteorol 96(1):33-62

Mandelbrot B (1983) The fractal geometry of nature, vol 173. W.H. Freeman \& Co Ltd, New York

Nepf HM (2012) Hydrodynamics of vegetated channels. J Hydraul Res 50(3):262-279

Placidi M, Ganapathisubramani B (2015) Effects of frontal and plan solidities on aerodynamic parameters and the roughness sublayer in turbulent boundary layers. J Fluid Mech 782:541-566

Placidi M, Ganapathisubramani B (2018) Turbulent flow over large roughness elements: effect of frontal and plan solidity on turbulence statistics and structure. Boundary-Layer Meteorol 167(1):99-121

Reynolds R, Castro I (2008) Measurements in an urban-type boundary layer. Exp Fluids 45(1):141-156

Schultz MP, Flack KA (2009) Turbulent boundary layers on a systematically varied rough wall. Phys Fluids 21(1):015104

Squire DT, Morrill-Winter C, Hutchins N, Schultz MP, Klewicki JC, Marusic I (2016) Comparison of turbulent boundary layers over smooth and rough surfaces up to high Reynolds numbers. J Fluid Mech 795:210-240

Vanderwel C, Ganapathisubramani B (2015) Effects of spanwise spacing on large-scale secondary flows in rough-wall turbulent boundary layers. J Fluid Mech 774:1-12

Xie ZT, Coceal O, Castro IP (2008) Large-eddy simulation of flows over random urban-like obstacles. Boundary-Layer Meteorol 129(1):1

Yang XIA, Sadique J, Mittal R, Meneveau C (2016) Exponential roughness layer and analytical model for turbulent boundary layer flow over rectangular-prism roughness elements. J Fluid Mech 789:127-165

Zhu X, Iungo GV, Leonardi S, Anderson W (2017) Parametric study of urban-like topographic statistical moments relevant to a priori modelling of bulk aerodynamic parameters. Boundary-Layer Meteorol 162(2):231-253

Publisher's Note Springer Nature remains neutral with regard to jurisdictional claims in published maps and institutional affiliations. 